\title{
32. PORE-WATER CHEMISTRY OF CARBONATE-RICH SEDIMENTS, LORD HOWE RISE, SOUTHWEST PACIFIC OCEAN ${ }^{1}$
}

\author{
Paul A. Baker, Department of Geology, Duke University²
}

\begin{abstract}
About 200 pore-water samples were recovered and analyzed from eight sites of DSDP Leg 90. At all sites on the carbonate-rich Lord Howe Rise, $\mathrm{Ca}^{2+}$ concentrations increase and $\mathrm{Mg}^{2+}$ concentrations decrease with increasing sub-bottom depth. The value of $\Delta \mathrm{Ca}^{2+} / \Delta \mathrm{Mg}^{2+}$ averages $-0.45 \mathrm{~mol} / \mathrm{mol}$ at these sites, an unusually small negative value in comparison with sites on basaltic crust. This supports the argument that the crust of the Lord Howe Rise is siliceous. $\mathrm{Li}^{+}$concentrations increase and $\mathrm{K}^{+}$concentrations decrease with depth. Both of these constituents are affected by reactions within the sediment column. Microbial sulfate reduction occurs to a small extent at all sites. In the upper $200 \mathrm{~m}$ of Site 594 on the south side of the Chatham Rise, sulfate reduction and alkalinity production are more pronounced.

Carbonate recrystallization is indicated by large increases in $\mathrm{Sr}^{2+}$ concentrations with depth at all sites. The exact value of the $\mathrm{Sr}^{2+}$ maximum at each site is determined by equilibrium with respect to celestite $\left(\mathrm{SrSO}_{4}\right)$. The greater the degree of microbial sulfate reduction, the higher is the pore water $\mathrm{Sr}^{2+}$ concentration. The diffusive flux of $\mathrm{Sr}^{2}$ out of the pore waters is consistent with an initial rate of carbonate recrystallization of $10 \% / \mathrm{m}$.y. This recrystallization must alter the isotopic and trace elemental compositions of some carbonate components.
\end{abstract}

\section{INTRODUCTION}

Leg 90 of the Deep Sea Drilling Project consisted of a north-south transect from $26^{\circ} \mathrm{S}$ to $41^{\circ} \mathrm{S}$ along the shallow (about $1000 \mathrm{~m}$ deep), carbonate-rich, Lord Howe Rise between New Caledonia, New Zealand, and Australia. An additional site, Site 594, was cored on the south side of the Chatham Rise at $46^{\circ} \mathrm{S}$ in a region presently characterized by more hemipelagic sedimentation. The major objective of this expedition was the recovery of complete Neogene sections for high-resolution paleoceanographic studies. We also obtained an atypically large number of interstitial water samples. Combined with detailed studies of sediment isotopic, chemical, and textural composition, the chemistry of Leg 90 pore waters provides important insights into the processes and rates of carbonate diagenesis.

\section{ANALYTICAL METHODS}

All interstitial waters analyzed in this study were obtained by shipboard extraction using the procedure and equipment described by Manheim and Sayles (1974). Extraction was effected as soon as possible after sediment recovery and was conducted at laboratory ambient temperature and pressure. Comparison of this extraction method with in situ sampling has established previously that temperature- and pressure-of-squeezing effects on the analyzed constituents are quite small (see Gieskes, 1973, 1974).

All analyses reported in this paper were carried out ashore. Prior to analysis, samples were stored unrefrigerated in either linear polyethylene bottles or sealed polyethylene or glass ampules. On the basis of previous experience, this storage procedure is acceptable for the reported constituents. Measurements that are in progress, but not published here, include the strontium and oxygen isotopic ratios of dissolved strontium and water, respectively.

Analytical methods for determination of chloride, calcium, magnesium, silica, alkalinity, and strontium are similar to those of Gieskes

\footnotetext{
${ }^{1}$ Kennett, J. P., von der Borch, C. C., et al., Init. Repts. DSDP, 90: Washington (U.S. Govt, Printing Office).

2 Address: Department of Geology, Duke University, Box 6729, College Station, Durham, NC 27708 .
}

(1974) and Gieskes and Lawrence (1976). Lithium, potassium, and sulfate methodologies are similar to those of Presley (1971).

\section{RESULTS AND DISCUSSION}

All the pore-water data are shown in Table 1 and Figures 1-6. The Lord Howe Rise sites (588, 590, 591, 592, and 593) and the lower $400 \mathrm{~m}$ of Site 594 display increasing $\mathrm{Ca}^{2+}$ and decreasing $\mathrm{Mg}^{2+}$ concentrations with increasing sub-bottom depth. The changes in $\mathrm{Ca}^{2+}$ and $\mathrm{Mg}^{2+}$ concentrations with depth at these sites are approximately linearly correlated (Fig. 7) with an average slope $\Delta \mathrm{Ca}^{2+} / \Delta \mathrm{Mg}^{2+}$ of about $-0.45 \mathrm{~mol} / \mathrm{mol}$. This slope is similar to those observed previously at drill sites overlying presumed siliceous basement rocks. Examples include (1) DSDP Sites 346 and 349 on the Jan-Mayen Ridge, north of Iceland, where $\Delta \mathrm{Ca}^{2+} / \Delta \mathrm{Mg}^{2+}$ is about -0.4 and -0.2 , respectively (Gieskes et al., 1978); (2) DSDP Site 116 on the Rockall Bank in the North Atlantic Ocean where $\Delta \mathrm{Ca}^{2+} / \Delta \mathrm{Mg}^{2+}$ is about -0.4 (Manheim et al., 1972); and (3) DSDP Site 237 on the Mascarene Ridge in the western Indian Ocean where $\Delta \mathrm{Ca}^{2+} / \Delta \mathrm{Mg}^{2+}$ is -0.57 (Sandstrom and Gieskes, 1974). By contrast, of those sites listed by McDuff (1981) in a compilation of DSDP sites displaying nonzero $\mathrm{Ca}^{2+} / \mathrm{Mg}^{2+}$ gradients and linear correlations of $\mathrm{Ca}^{2+}$ versus $\mathrm{Mg}^{2+}$, not a single one overlying basaltic basement had a value of $-\Delta \mathrm{Ca}^{2+} / \Delta \mathrm{Mg}^{2+}$ as low as any of these on siliceous crust. A typical value of $\Delta \mathrm{Ca}^{2+} / \Delta \mathrm{Mg}^{2+}$ for sites on basaltic crust is -1.5 (McDuff, 1981). It has previously been shown (McDuff and Gieskes, 1976, McDuff, 1981) that linear correlation of $\mathrm{Ca}^{2+}$ and $\mathrm{Mg}^{2+}$ is usually indicative of conservative chemical behavior in the sediment column. As basement rocks alter, they take up magnesium from the pore waters and release calcium to the pore waters. Apparently, as basement rocks of the Lord Howe Rise alter, they take up more magnesium and release less calcium than do basaltic basement rocks. The implication of these observations for Leg 90 sites is sup- 
Table 1. Interstitial water concentration data.

\begin{tabular}{|c|c|c|c|c|c|c|c|c|c|}
\hline $\begin{array}{l}\text { Core-Section } \\
\text { (interval in cm) }\end{array}$ & $\begin{array}{l}\text { Depth } \\
\text { (m) }\end{array}$ & $\begin{array}{l}\mathrm{Ca}^{2+} \\
(\mathrm{mM})\end{array}$ & $\begin{array}{l}\mathrm{Mg}^{2+} \\
(\mathrm{mM})\end{array}$ & $\begin{array}{l}\mathrm{Sr}^{2+} \\
(\mathrm{mM})\end{array}$ & $\begin{array}{l}\mathrm{K}^{+} \\
(\mathrm{mM})\end{array}$ & $\begin{array}{l}\mathrm{Li}^{+} \\
(\mu \mathrm{M})\end{array}$ & $\begin{array}{l}\mathrm{Sio}_{2} \\
(\mu \mathrm{M})\end{array}$ & $\begin{array}{l}\text { Alkalinity } \\
\text { (meq/l) }\end{array}$ & $\begin{array}{l}\mathrm{SO}_{4}^{2-} \\
(\mathrm{mM})\end{array}$ \\
\hline \multicolumn{10}{|l|}{ Site 587} \\
\hline $2-3,144-150$ & 7.57 & 9.03 & 54.66 & 0.147 & 10.49 & - & 186 & 3.64 & 26.7 \\
\hline $3-6,0-6$ & 20.23 & 8.79 & 55.40 & 0.287 & 11.54 & - & 214 & 3.51 & 28.0 \\
\hline $5-3,144-150$ & 36.37 & 9.30 & 53.98 & 0.293 & 11.54 & - & 147 & 3.36 & 27.4 \\
\hline $7-3,144-150$ & 55.57 & 9.74 & 53.54 & 0.306 & 10.68 & - & - & 3.28 & 25.7 \\
\hline $9-1,144-150$ & 71.77 & 9.83 & 53.74 & 0.363 & 11.75 & - & - & 3.09 & - \\
\hline \multicolumn{10}{|l|}{ Hole 588} \\
\hline $1-3,144-150$ & 4.47 & 10.63 & 51.03 & - & 10.49 & 31.0 & 220 & 2.84 & 25.0 \\
\hline $2-5,144-150$ & 13.07 & 10.47 & 51.23 & 0.249 & 11.76 & 32.8 & 190 & 2.74 & 26.1 \\
\hline $3-4,144-150$ & 21.17 & 10.51 & 51.03 & 0.300 & 11.50 & 34.2 & 169 & 2.83 & 25.7 \\
\hline $4-4,144-150$ & 30.77 & 10.72 & 51.07 & 0.395 & 11.54 & 36.3 & 149 & 2.77 & 25.8 \\
\hline $5-4,144-150$ & 40.37 & 11.01 & 50.56 & 0.446 & 10.91 & 37.0 & 158 & 2.84 & 25.4 \\
\hline $6-4,144-150$ & 49.97 & 11.20 & 49.95 & 0.523 & 10.75 & 39.0 & 156 & 3.04 & 24.7 \\
\hline $7-4,144-150$ & 59.57 & 11.15 & 49.97 & 0.561 & 10.75 & 39.6 & 141 & - & \\
\hline $9-4,144-150$ & 78.77 & 11.76 & 49.25 & 0.644 & 10.18 & 41.4 & 152 & 3.06 & 23.3 \\
\hline $10-4,144-150$ & 88.37 & 11.93 & 47.79 & 0.638 & 10.31 & 42.8 & 164 & 2.78 & 24.7 \\
\hline $11-4,144-150$ & 97.97 & 12.10 & 48.04 & 0.701 & 10.23 & 43.6 & 160 & 2.97 & 23.7 \\
\hline $12-4,144-150$ & 107.57 & 12.11 & 48.15 & 0.714 & 10.16 & 44.3 & 165 & 2.95 & 23.6 \\
\hline $13-4,144-150$ & 117.47 & 12.47 & 47.19 & 0.746 & 10.44 & 46.6 & 201 & 2.90 & 23.6 \\
\hline $14-3,144-150$ & 125.27 & 12.71 & 46.45 & 0.708 & - & 48.1 & 211 & 2.91 & 23.4 \\
\hline $15-3,144-150$ & 134.87 & 12.77 & 46.33 & 0.714 & 10.01 & 47.8 & 182 & 2.86 & 23.3 \\
\hline $16-3,144-150$ & 144.47 & 12.97 & 46.25 & 0.708 & 10.01 & 49.1 & 210 & 2.79 & 22.9 \\
\hline $17-3,144-150$ & 154.07 & 13.17 & 45.80 & 0.708 & 10.12 & 51.1 & 226 & 2.74 & 23.3 \\
\hline $18-5,144-150$ & 166.67 & 13.38 & 46.75 & 0.714 & 10.01 & 55.7 & 233 & 2.72 & 23.3 \\
\hline $19-5,144-150$ & 176.27 & 13.23 & 45.59 & 0.733 & 9.79 & 54.2 & 226 & 2.71 & 22.6 \\
\hline $20-3,144-150$ & 182.87 & 13.32 & 44.58 & 0.663 & 9.62 & 55.0 & 222 & 2.75 & 22.9 \\
\hline $21-4,144-150$ & 193.97 & 13.36 & 44.91 & 0.689 & 9.63 & 58.7 & 228 & 2.50 & 22.3 \\
\hline $22-4,144-150$ & 203.57 & 13.58 & 44.43 & 0.682 & 10.16 & 59.6 & 324 & 2.22 & 22.9 \\
\hline $23-3,144-150$ & 211.67 & 13.42 & 43.57 & 0.676 & 10.16 & 61.7 & 258 & 1.98 & 23.4 \\
\hline $24-3,144-150$ & 221.27 & 11.79 & 45.57 & 0.689 & 9.96 & 63.0 & 246 & - & 23.6 \\
\hline $25-3,144-150$ & 230.87 & 11.81 & 45.57 & 0.669 & 10.12 & 66.2 & 284 & 1.90 & 22.9 \\
\hline
\end{tabular}

Hole 588A

$\begin{array}{llllllllll}1-4,144-150 & 241.97 & 13.62 & 37.42 & 0.701 & 10.49 & 69.7 & 150 & 1.67 & 22.7 \\ 3-2,144-150 & 253.57 & 14.28 & 42.30 & 0.714 & 10.15 & 73.9 & 158 & 1.99 & 22.6 \\ 5-2,144-150 & 263.57 & 14.13 & 42.33 & 0.695 & 10.03 & 77.1 & 158 & 1.71 & 21.7 \\ 7-2,144-150 & 273.57 & 14.31 & 42.71 & 0.720 & 9.24 & 80.0 & 169 & 1.78 & 21.6 \\ 9-2,144-150 & 283.57 & 14.20 & 42.00 & 0.708 & - & 84.8 & - & - & 22.9 \\ 12-2,140-150 & 298.55 & 14.31 & 39.95 & 0.701 & 10.35 & 84.5 & 166 & 0.46 & 22.1 \\ 14-1,140-150 & 307.05 & 14.62 & 41.37 & 0.708 & 10.18 & 84.3 & 169 & 0.97 & 22.0\end{array}$

\section{Hole 588C}

$1-5,140-150$
$3-3,140-150$
$5-4,140-150$
$7-2,140-150$
$9-5,140-150$
$11-1,140-150$
$13-3,140-150$
$15-4,140-150$
$17-3,140-150$

313.15

$350.05 \quad-$

$366.25 \quad-\quad-\quad 0.740$

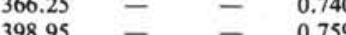

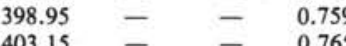

$403.15 \quad-\quad-\quad 0.765$

\begin{tabular}{l}
$425.35 \quad-\quad-\quad 0.759$ \\
\hline
\end{tabular}

$\begin{array}{llll}446.05 & - & - & 0.759\end{array}$

$\begin{array}{llll}463.75 & - & - & 0.822 \\ & - & 0.746\end{array}$
$9.64 \quad 92.0 \quad 10$

10.2399.

$9.84 \quad 102.2$

$9.74 \quad 105.2$

$9.41 \quad 118.5$

$9.79 \quad 124.3$

$9.05 \quad 115.5$
$8.67 \quad 107.0$

Site 589
$1-4,140-150$
$2-5,144-150$

$3-5,144-150$

$4-4,144-150$
5.95

$24.37 \quad 10.70$

32.47
$11.22 \quad 47$

Hole 590

$1-3,144-150$

$2-3,144-150$

$\begin{array}{rrrr}4.47 & 10.66 & 50.89 & 0.172 \\ 11.47 & 10.85 & 49.59 & 0.300\end{array}$

$3-3,144-150$

21.07

$\begin{array}{lllll}10.85 & 49.59 & 0.172 & 11.39 & 33.2 \\ 11.23 & 38.1\end{array}$

$\begin{array}{lll}10.78 & 50.90 & 0.27\end{array}$
$0.191 \quad 12.38$

$402 \quad 12.62$

\begin{tabular}{ll}
.402 & 12.62 \\
\hline & 12.38
\end{tabular}
$0.300 \quad 11.25$
$34.9 \quad 252$

$36.0 \quad 277$

$39.2 \quad 261$

$41.0 \quad 233$
Hole 590A

$1-3,144-150$

$2-3,144-150$

$3-3,144-150$

4-5, 144-150

$5-3,144-150$

\begin{tabular}{lllllllll}
30.65 & 11.23 & 47.73 & 0.504 & 11.17 & 38.6 & 241 & 4.84 & 21.7 \\
40.27 & 11.39 & 46.49 & 0.587 & 11.02 & 40.8 & 188 & 4.52 & 22.6 \\
49.87 & 11.48 & 45.13 & 0.657 & 11.02 & 41.4 & 182 & 4.02 & 21.1 \\
62.47 & 11.84 & 44.49 & 0.797 & 11.20 & 42.8 & 177 & 4.30 & 20.7 \\
69.07 & 12.29 & 44.07 & 0.759 & 10.69 & 42.8 & 175 & 3.77 & 19.7 \\
\hline
\end{tabular}


Table 1. (Continued).

\begin{tabular}{|c|c|c|c|c|c|c|c|c|c|}
\hline $\begin{array}{c}\text { Core-Section } \\
\text { (interval in cm) }\end{array}$ & $\begin{array}{l}\text { Depth } \\
\text { (m) }\end{array}$ & $\begin{array}{l}\mathrm{Ca}^{2+} \\
(\mathrm{mM})\end{array}$ & $\begin{array}{l}\mathrm{Mg}^{2+} \\
(\mathrm{mM})\end{array}$ & $\begin{array}{l}\mathrm{Sr}^{2+} \\
(\mathrm{mM})\end{array}$ & $\underset{(\mathrm{mM})}{\mathrm{K}^{+}}$ & $\begin{array}{l}\mathrm{Li}^{+} \\
(\mu \mathrm{M})\end{array}$ & $\begin{array}{l}\mathrm{Sio}_{2} \\
(\mu \mathrm{M})\end{array}$ & $\begin{array}{c}\text { Alkalinity } \\
\text { (meq/l) }\end{array}$ & $\begin{array}{l}\mathrm{SO}_{4}^{2-} \\
(\mathrm{mM})\end{array}$ \\
\hline \multicolumn{10}{|l|}{ Hole 590A (cont.) } \\
\hline $6-3,144-150$ & 78.67 & 12.93 & 43.31 & 0.848 & - & - & 186 & 3.90 & \\
\hline $7-3,144-150$ & 88.27 & 12.78 & 43.12 & 0.880 & 10.71 & 42.6 & 177 & 4.56 & 18.4 \\
\hline $8-3,144-150$ & 97.87 & 12.66 & 41.25 & 0.854 & 11.09 & 43.6 & 181 & 3.63 & 19.1 \\
\hline $10-3,144-150$ & 117.07 & 13.64 & 40.17 & 0.842 & 10.71 & 41.6 & 192 & 2.96 & 18.3 \\
\hline $11-3,144-150$ & 126.67 & 13.81 & 40.21 & 0.880 & - & 40.7 & 194 & 2.53 & - \\
\hline $12-3,144-150$ & 136.27 & 13.76 & 38.84 & 0.874 & 10.61 & 40.8 & 201 & 2.95 & 17.4 \\
\hline $13-3,144-150$ & 145.87 & 14.54 & 38.58 & 0.880 & 10.73 & 40.5 & 214 & 3.36 & 17.3 \\
\hline $14-3,144-150$ & 155.47 & 13.99 & 38.63 & 0.854 & 9.88 & 39.7 & 216 & 2.46 & 17.3 \\
\hline $15-3,144-150$ & 165.07 & 14.71 & 36.73 & 0.893 & 10.31 & 40.2 & 235 & - & 16.1 \\
\hline $16-3,144-150$ & 174.67 & 14.89 & 37.72 & 0.880 & 10.27 & 39.7 & 246 & 3.65 & 16.4 \\
\hline $17-3,144-150$ & 184.27 & 14.75 & 36.11 & 0.880 & 10.42 & 39.5 & 260 & 2.89 & 15.7 \\
\hline $18-3,144-150$ & 193.87 & 15.50 & 37.10 & 0.893 & - & 39.8 & 284 & 3.09 & 16.6 \\
\hline $19-3,144-150$ & 203.47 & 15.37 & 34.96 & 0.905 & - & 45.6 & 310 & 2.19 & 16.6 \\
\hline $20-3,144-150$ & 213.07 & 15.72 & 37.00 & 0.899 & 10.06 & 47.2 & 337 & 2.38 & 15.1 \\
\hline $21-3,144-150$ & 222.67 & 15.70 & 34.95 & 0.905 & 10.66 & 42.1 & 395 & 2.17 & 14.4 \\
\hline $23-3,144-150$ & 241.87 & 16.17 & 33.88 & 0.842 & 10.49 & 46.0 & 504 & 2.04 & 15.4 \\
\hline $24-3,144-150$ & 251.47 & 16.64 & 33.92 & 0.848 & 10.71 & 47.7 & 609 & - & 15.6 \\
\hline $25-3,144-150$ & 261.07 & 16.37 & 34.06 & 0.842 & 10.39 & 48.9 & 781 & 2.17 & 15.9 \\
\hline $26-3,144-150$ & 270.67 & 17.27 & 33.56 & 0.822 & 10.07 & 51.0 & 899 & - & 16.0 \\
\hline $27-2,144-150$ & 278.77 & 16.68 & 33.63 & 0.829 & 9.92 & 53.7 & 976 & 1.99 & 14.9 \\
\hline \multicolumn{10}{|l|}{ Hole 590B } \\
\hline $33-5,140-150$ & 304.95 & 17.52 & 33.36 & 0.842 & 10.30 & 65.8 & 1035 & - & 16. \\
\hline $35-4,140-150$ & 322.65 & 18.23 & 32.59 & 0.829 & 10.23 & 79.6 & 1060 & - & 15. \\
\hline $37-4,140-150$ & 341.85 & 18.72 & 31.89 & 0.867 & 10.37 & 92.4 & 1135 & 2.12 & 15. \\
\hline $39-5,140-150$ & 362.55 & 19.73 & 32.57 & 0.854 & 10.30 & 104.4 & 840 & - & 16. \\
\hline $41-5,140-150$ & 381.75 & 19.98 & 32.38 & 0.867 & 9.92 & 115.5 & 622 & 2.22 & 17. \\
\hline $43-3,140-150$ & 397.95 & 19.45 & 31.69 & 0.848 & 10.01 & 125.2 & 574 & - & 16.3 \\
\hline $45-4,140-150$ & 418.65 & 20.44 & 31.65 & 0.886 & 9.77 & 137.5 & 672 & - & 16. \\
\hline $47-6,140-150$ & 440.85 & 20.24 & 31.53 & 0.867 & 9.73 & 148.7 & 647 & 1.80 & 16. \\
\hline $49-4,1401-50$ & 457.05 & 19.28 & 33.06 & 0.791 & 9.59 & 143.2 & 357 & - & 17. \\
\hline $51-3,140-150$ & 474.75 & 19.52 & 33.37 & 0.835 & 9.88 & 135.2 & 361 & 1.91 & 17. \\
\hline $53-4,1401-50$ & 495.45 & 19.42 & 34.06 & 0.822 & 9.76 & 131.7 & 378 & - & 18. \\
\hline
\end{tabular}

Hole 591

$\begin{array}{lrllllllll}2-3,144-150 & 7.87 & 10.53 & 50.88 & 0.198 & 11.95 & 34.5 & 354 & 4.44 & 24.3 \\ 4-3,144-150 & 27.07 & 11.35 & 49.94 & 0.389 & 11.81 & 33.4 & 492 & 3.21 & 22.7 \\ 6-3,144-150 & 46.27 & 11.28 & 44.81 & 0.548 & 12.76 & 35.2 & 497 & 3.87 & 21.4 \\ 8-3,144-150 & 65.47 & 12.24 & 45.15 & 0.759 & 11.90 & 34.6 & 583 & 4.68 & 20.7 \\ 10-3,144-150 & 84.67 & 13.20 & 41.89 & 0.829 & 12.00 & 33.4 & 672 & 5.05 & 19.6 \\ 12-3,144-150 & 103.87 & 12.98 & 40.59 & 0.880 & 11.40 & 30.5 & 700 & 4.32 & 18.7 \\ 14-2,144-150 & 121.27 & 13.27 & 39.70 & 0.925 & 12.14 & 29.6 & 831 & 3.30 & 18.9 \\ 16-4,144-150 & 142.87 & 14.04 & 37.63 & 0.931 & 12.31 & 27.0 & 935 & 2.52 & 17.3 \\ 18-4,144-150 & 161.47 & 15.29 & 36.52 & 0.925 & 12.07 & 27.1 & 950 & 2.64 & 18.0 \\ 20-2,144-150 & 177.27 & 14.60 & 36.16 & 0.944 & 12.18 & 25.3 & 988 & - & 17.9 \\ 22-4,144-150 & 199.27 & 15.27 & 36.15 & 0.918 & 12.09 & 24.9 & 1001 & 2.58 & 17.0 \\ 24-3,144-150 & 216.57 & 15.99 & 34.39 & 0.931 & 12.18 & 25.5 & 1026 & 2.34 & 16.9 \\ 26-3,144-150 & 235.57 & 15.31 & 34.01 & 0.969 & 12.03 & 28.9 & 1236 & - & 15.7 \\ 28-3,144-150 & 254.77 & 16.53 & 32.14 & 0.969 & 12.04 & 27.3 & 1048 & - & 16.9 \\ 30-2,144-150 & 272.27 & 17.74 & 31.59 & 0.950 & 11.97 & 26.6 & 1073 & 2.21 & 16.7\end{array}$

Hole 591B

$\begin{array}{lrrrrrrrrr}2-3,140-150 & 284.67 & 18.64 & 31.74 & 0.925 & 12.06 & 29.6 & 1056 & 1.84 & 17.0 \\ 4-3,140-150 & 303.85 & 18.36 & 31.56 & 0.931 & 11.54 & 28.7 & 1037 & - & - \\ 6-3,140-150 & 322.65 & 18.61 & 31.33 & 0.912 & 11.87 & 31.7 & 1071 & 1.85 & - \\ 10-2,140-150 & 359.35 & 19.13 & 31.59 & 0.905 & 11.23 & 42.1 & 1005 & - & - \\ 12-1,140-150 & 377.05 & 19.44 & 29.79 & 0.912 & 11.87 & 46.2 & 1039 & 2.15 & - \\ 14-1,140-150 & 396.25 & 19.96 & 31.02 & 0.899 & 11.45 & 52.2 & 1082 & 1.86 & - \\ 18-3,140-150 & 437.65 & 20.32 & 30.07 & 0.918 & 11.72 & 66.1 & 980 & - & - \\ 20-3,140-150 & 456.85 & 20.02 & 29.09 & 0.889 & - & 70.9 & 1012 & 2.26 & - \\ 22-4,140-150 & 477.55 & 19.63 & 28.91 & 0.880 & 12.10 & 76.1 & 990 & 2.43 & - \\ 24-4,140-150 & 496.75 & 19.58 & 29.42 & 0.867 & 11.85 & 76.4 & - & 2.23 & - \\ & & & & & & & & & \\ & & & & & & & & & \\ 2-3,144-150 & 8.97 & 11.14 & 50.72 & 0.185 & 11.16 & 33.9 & - & 2.85 & 27.3 \\ 4-4,144-150 & 29.67 & 11.39 & - & 0.370 & 11.26 & - & 207 & 3.39 & 26.0 \\ 6-3,144-150 & 47.37 & 11.89 & 47.56 & 0.497 & 10.67 & 40.1 & - & 3.32 & 24.9 \\ 8-3,144-150 & 66.57 & 12.26 & 46.91 & 0.580 & 10.49 & 41.4 & 184 & 3.90 & 24.3 \\ 10-3,144-150 & 85.77 & 13.15 & 45.38 & 0.676 & 10.69 & 44.3 & 186 & 3.84 & 22.9 \\ 12-3,144-150 & 104.97 & 13.23 & 45.58 & 0.752 & 10.12 & 46.1 & 190 & 4.00 & 22.4 \\ 14-3,144-150 & 124.17 & 13.65 & 44.25 & 0.769 & 9.21 & 48.3 & 199 & 4.12 & 22.7 \\ 16-3,144-150 & 143.37 & 14.15 & 43.99 & 0.784 & 9.81 & 49.2 & 213 & 4.04 & 21.6\end{array}$


Table 1. (Continued).

\begin{tabular}{|c|c|c|c|c|c|c|c|c|c|}
\hline $\begin{array}{l}\text { Core-Section } \\
\text { (interval in cm) }\end{array}$ & $\begin{array}{l}\text { Depth } \\
\text { (m) }\end{array}$ & $\begin{array}{l}\mathrm{Ca}^{2+} \\
(\mathrm{mM})\end{array}$ & $\begin{array}{l}\mathrm{Mg}^{2+} \\
(\mathrm{mM})\end{array}$ & $\begin{array}{l}\mathrm{Sr}^{2+} \\
(\mathrm{mM})\end{array}$ & $\underset{(\mathrm{mM})}{\mathrm{K}^{+}}$ & $\begin{array}{l}\mathrm{Li}^{+} \\
(\mu \mathrm{M})\end{array}$ & $\begin{array}{l}\mathrm{Sio}_{2} \\
(\mu \mathrm{M})\end{array}$ & $\begin{array}{l}\text { Alkalinity } \\
\text { (meq/l) }\end{array}$ & $\begin{array}{l}\mathrm{SO}_{4}^{2-} \\
(\mathrm{mM})\end{array}$ \\
\hline \multicolumn{10}{|l|}{ Hole 592 (cont.) } \\
\hline $18-3,144-150$ & 162.57 & 14.50 & 43.07 & 0.784 & 8.61 & 50.9 & 222 & 3.92 & 20.9 \\
\hline $20-3,144-150$ & 181.77 & 15.00 & 42.65 & 0.740 & 9.36 & 51.9 & 224 & 3.99 & 21.1 \\
\hline $22-3,144-150$ & 200.97 & 15.38 & 41.36 & 0.778 & 9.58 & 54.7 & 199 & 3.98 & 20.9 \\
\hline $24-3,144-150$ & 220.17 & 15.70 & 40.90 & 0.784 & 9.81 & 57.5 & 190 & 3.63 & 20.3 \\
\hline $26-3,144-150$ & 239.37 & 15.86 & 40.67 & 0.797 & 9.05 & 58.6 & 196 & 3.65 & 20.9 \\
\hline $28-3,144-150$ & 258.57 & 15.66 & 40.75 & 0.784 & 9.47 & 60.7 & 211 & 3.28 & 20.7 \\
\hline $31-3,140-150$ & 287.35 & 16.10 & 39.60 & 0.791 & 8.61 & 62.7 & 286 & 2.71 & 19.7 \\
\hline $33-3,140-150$ & 306.55 & 16.71 & 38.54 & 0.771 & 8.93 & 65.2 & 465 & 3.02 & 20.1 \\
\hline $35-3,140-150$ & 325.75 & 17.16 & 38.08 & 0.791 & 8.35 & 66.4 & - & - & 19.7 \\
\hline $37-3,140-150$ & 344.95 & 17.20 & 38.04 & 0.797 & 8.49 & 77.4 & 925 & 3.29 & 19.1 \\
\hline $39-3,140-150$ & 364.15 & 17.34 & 37.44 & 0.797 & 8.37 & 80.0 & 1033 & 3.27 & 18.7 \\
\hline $41-3,140-150$ & 383.35 & 17.54 & 36.86 & 0.797 & 8.30 & 83.0 & 946 & 3.18 & 18.9 \\
\hline \multicolumn{10}{|l|}{ Site 593} \\
\hline $1-4,144-150$ & 5.97 & 10.56 & 51.96 & 0.166 & 11.07 & 31.6 & - & 3.42 & 27.0 \\
\hline $4-3,144-150$ & 28.77 & 10.54 & 51.42 & 0.236 & 11.74 & 29.2 & 405 & 3.34 & 27.1 \\
\hline $6-3,144-150$ & 47.97 & 10.68 & 49.99 & 0.338 & 11.30 & 32.6 & - & 3.34 & 26.4 \\
\hline $8-1,144-150$ & 62.37 & 10.74 & 49.90 & 0.389 & 10.92 & 35.8 & 182 & 3.36 & 25.0 \\
\hline $12-3,144-150$ & 105.57 & 11.24 & 48.99 & 0.529 & 10.81 & 38.3 & - & 4.12 & 24.9 \\
\hline $14-3,144-150$ & 124.77 & 11.66 & 48.69 & 0.671 & 10.08 & 39.4 & - & 3.35 & 25.0 \\
\hline $16-3,144-150$ & 143.17 & 11.62 & 48.23 & 0.631 & 9.95 & 39.6 & 177 & 3.94 & 25.0 \\
\hline $18-3,144-150$ & 163.17 & 11.74 & 47.97 & 0.657 & 9.91 & 41.0 & - & 3.54 & 24.1 \\
\hline $20-3,144-150$ & 182.37 & 12.18 & 46.37 & - & 10.12 & 42.5 & - & - & 24.6 \\
\hline $22-3,144-150$ & 201.57 & 12.15 & 46.82 & 0.701 & 9.33 & 45.5 & 254 & 3.55 & 23.9 \\
\hline $24-3,144-150$ & 220.77 & 12.64 & 46.06 & 0.733 & 9.44 & 45.1 & 241 & 3.78 & 24.3 \\
\hline $26-3,144-150$ & 239.97 & 12.79 & 45.91 & 0.720 & 9.65 & 47.3 & 203 & 3.14 & 23.1 \\
\hline $28-3,144-150$ & 259.17 & 13.17 & 44.02 & 0.733 & 9.37 & 49.8 & 190 & 2.73 & 22.6 \\
\hline $30-3,144-150$ & 278.37 & 13.44 & 43.04 & 0.752 & 9.35 & 51.5 & - & - & 23.1 \\
\hline $32-2,144-150$ & 296.07 & 13.84 & 42.68 & 0.695 & 9.16 & 53.1 & - & 2.99 & 23.4 \\
\hline $34-2,144-150$ & 315.27 & 13.94 & 42.67 & 0.759 & 9.16 & 54.3 & 171 & 2.81 & 22.4 \\
\hline $36-2,144-150$ & 334.47 & 14.16 & 42.93 & 0.714 & 9.09 & 57.3 & - & 2.28 & 22.4 \\
\hline $38-3,144-150$ & 355.17 & 14.31 & 41.73 & 0.752 & 9.02 & 58.2 & 162 & 2.93 & 21.4 \\
\hline $40-3,144-150$ & 374.37 & 14.77 & 40.57 & 0.733 & 8.51 & 61.0 & - & 2.91 & 21.6 \\
\hline $42-3,144-150$ & 393.57 & 14.81 & 39.48 & 0.759 & - & 62.8 & - & 2.64 & 21.0 \\
\hline $44-3,144-150$ & 412.77 & 15.46 & 38.69 & 0.765 & 8.30 & 68.4 & - & 2.52 & 21.9 \\
\hline $46-3,144-150$ & 431.97 & 15.86 & 37.65 & 0.771 & 8.49 & 71.6 & - & 2.44 & 21.4 \\
\hline $47-3,144-150$ & 441.57 & 15.49 & - & 0.746 & 9.28 & 71.9 & - & 1.99 & 21.9 \\
\hline $48-3,144-150$ & 451.17 & 15.71 & 37.24 & 0.733 & 8.45 & 69.7 & 186 & - & 22.1 \\
\hline $50-3,144-150$ & 470.37 & 15.69 & 36.60 & 0.759 & 9.21 & 95.5 & - & 2.08 & 21.3 \\
\hline $54-2,144-150$ & 507.27 & 16.71 & 34.13 & 0.708 & 8.72 & 83.8 & 459 & - & 20.9 \\
\hline $56-3,144-150$ & 527.97 & 17.12 & 33.50 & 0.746 & - & - & - & - & 20.6 \\
\hline $58-1,140-150$ & 544.15 & 16.02 & 34.81 & 0.714 & 8.84 & 88.3 & 425 & 2.14 & 21.4 \\
\hline $60-3,144-150$ & 566.37 & 15.14 & 30.16 & 0.689 & 7.79 & 179.5 & - & - & - \\
\hline \multicolumn{10}{|l|}{ Hole 594} \\
\hline $2-3,144-150$ & 10.37 & 6.45 & 51.80 & 0.070 & 11.52 & 32.3 & 689 & 9.57 & 19.6 \\
\hline $4-3,144-150$ & 29.57 & 6.46 & 47.66 & 0.064 & 12.16 & 34.2 & 761 & 9.83 & 15.3 \\
\hline $6-3,144-150$ & 48.77 & 5.83 & 45.45 & 0.070 & 11.54 & 36.1 & 859 & 14.94 & 10.1 \\
\hline $8-3,144-150$ & 64.97 & 3.96 & 44.53 & 0.102 & 12.18 & - & - & - & 9.0 \\
\hline $10-1,144-150$ & 84.17 & 5.03 & 41.96 & 0.134 & 10.85 & 43.7 & 808 & 13.06 & - \\
\hline $12-3,144-150$ & 106.37 & 5.14 & 41.84 & 0.140 & 11.63 & 47.7 & 819 & 9.38 & 10.6 \\
\hline $15-3,144-150$ & 135.17 & 5.90 & 43.82 & 0.191 & 11.04 & 40.4 & 931 & 9.28 & 13.3 \\
\hline $18-3,144-150$ & 163.97 & 8.01 & 44.03 & 0.306 & 10.88 & - & - & 7.80 & 16.3 \\
\hline $20-3,144-150$ & 183.17 & 9.24 & 43.17 & 0.338 & 10.67 & 31.5 & 954 & 5.62 & 18.0 \\
\hline $24-3,144-150$ & 221.57 & 12.44 & 41.07 & 0.555 & 9.01 & 27.6 & 1039 & 4.84 & 18.9 \\
\hline $26-3,144-150$ & 240.77 & 14.17 & 39.63 & 0.620 & 9.51 & - & - & - & 20.1 \\
\hline $28-3,144-150$ & 259.97 & 15.02 & 38.88 & 0.657 & 9.70 & 24.8 & 1137 & 2.48 & 19.4 \\
\hline $30-3,144-150$ & 279.17 & 16.34 & 38.62 & 0.720 & 9.23 & - & 1043 & 2.97 & 20.3 \\
\hline $34-3,144-150$ & 316.57 & 17.83 & 36.33 & 0.759 & 9.46 & - & - & - & 19.1 \\
\hline $36-3,144-150$ & 335.77 & 18.31 & 35.25 & 0.797 & 7.79 & 24.8 & - & 2.44 & 19.0 \\
\hline $38-2,144-150$ & 353.47 & 19.31 & 33.98 & 0.810 & 8.23 & 26.1 & - & 2.23 & 19.3 \\
\hline $40-3,144-150$ & 374.17 & 20.05 & 32.34 & 0.816 & 8.54 & 27.9 & 1205 & - & 19.6 \\
\hline $42-3,144-150$ & 394.37 & 20.49 & 32.66 & 0.835 & 8.65 & 30.8 & - & 3.16 & 19.0 \\
\hline $44-3,144-150$ & 414.57 & 21.44 & 29.63 & 0.861 & 8.16 & 35.5 & 1139 & 3.22 & 18.3 \\
\hline $46-1,144-150$ & 429.77 & 22.00 & 29.07 & 0.861 & 6.58 & 39.8 & 1173 & 3.18 & 19.1 \\
\hline $48-2,144-150$ & 450.47 & 22.58 & 28.94 & 0.867 & 7.24 & 43.7 & 1196 & 3.06 & 18.9 \\
\hline \multicolumn{10}{|l|}{ Hole 594A } \\
\hline $11-3,144-150$ & 202.37 & 12.08 & 40.89 & 0.478 & - & 30.2 & 908 & 3.11 & 17.6 \\
\hline $12-1,138-144$ & 496.91 & 24.82 & 24.59 & 0.931 & 7.10 & 52.1 & 1364 & 2.99 & 17.1 \\
\hline $14-3,140-150$ & 519.15 & 24.76 & 25.49 & 0.931 & 6.82 & 56.5 & 1340 & 2.68 & 17.0 \\
\hline $16-3,140-150$ & 538.35 & 24.56 & 25.43 & 0.937 & - & 59.7 & 1132 & 2.82 & 17.6 \\
\hline $19-1,140-150$ & 564.15 & 25.23 & 23.83 & 0.976 & 6.44 & 63.1 & 795 & 1.99 & 16.6 \\
\hline
\end{tabular}


Table 1. (Continued).

\begin{tabular}{rccccccccc}
\hline $\begin{array}{c}\text { Core-Section } \\
\text { (interval in } \mathrm{cm})\end{array}$ & $\begin{array}{c}\text { Depth } \\
(\mathrm{m})\end{array}$ & $\begin{array}{c}\mathrm{Ca}^{2+} \\
(\mathrm{mM})\end{array}$ & $\begin{array}{c}\mathrm{Mg}^{2+} \\
(\mathrm{mM})\end{array}$ & $\begin{array}{c}\mathrm{Sr}^{2+} \\
(\mathrm{mM})\end{array}$ & $\begin{array}{c}\mathrm{K}^{+} \\
(\mathrm{mM})\end{array}$ & $\begin{array}{c}\mathrm{Li}^{+} \\
(\mu \mathrm{M})\end{array}$ & $\begin{array}{c}\mathrm{Sio}_{2} \\
(\mu \mathrm{M})\end{array}$ & $\begin{array}{c}\text { Alkalinity } \\
(\mathrm{meq} / \mathrm{l})\end{array}$ & $\begin{array}{c}\mathrm{SO}_{4}^{2-} \\
(\mathrm{mM})\end{array}$ \\
\hline Hole 594A (cont.) & & & & & & & & & \\
$21-1,140-150$ & 583.35 & 24.94 & 23.10 & 0.956 & 6.34 & 68.1 & - & - & 15.9 \\
$23-2,140-150$ & 604.05 & - & - & - & - & - & - & - & - \\
$25-2,140-150$ & 623.25 & 25.11 & 23.05 & 0.963 & 5.48 & 69.0 & 692 & 1.81 & 16.1 \\
\hline
\end{tabular}

Note: Dashes indicate that no analysis was performed.

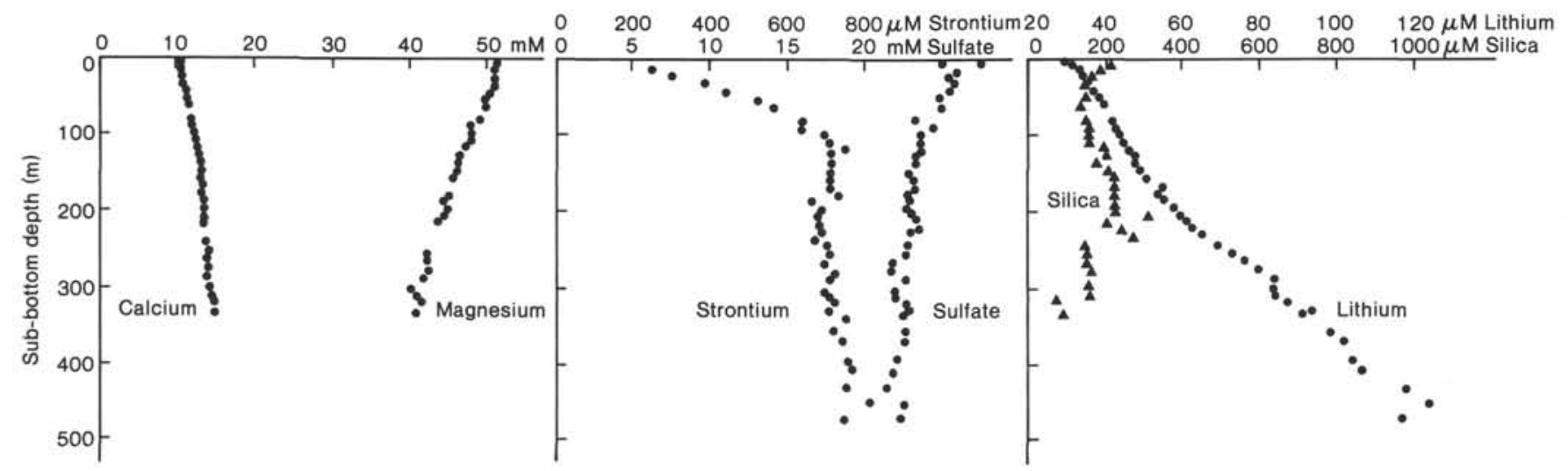

Figure 1. Pore-water concentrations of calcium, magnesium, strontium, sulfate, lithium, and silica as a function of sub-bottom depth of Site 588.

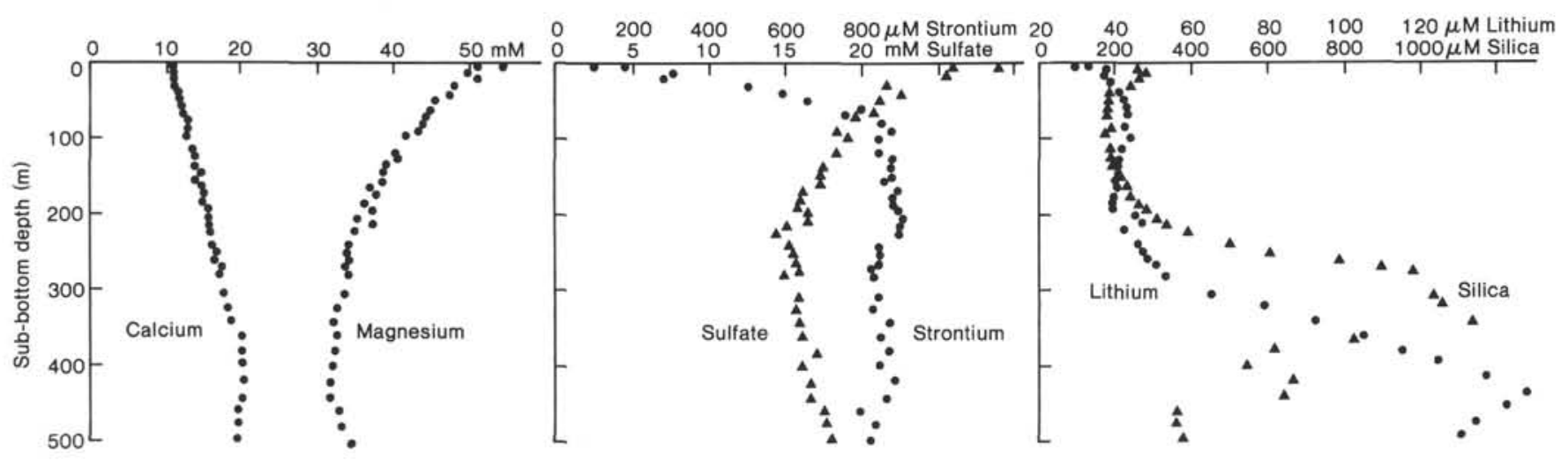

Figure 2. Pore-water concentrations of calcium, magnesium, strontium, sulfate, lithium, and silica as a function of sub-bottom depth at Site 590.

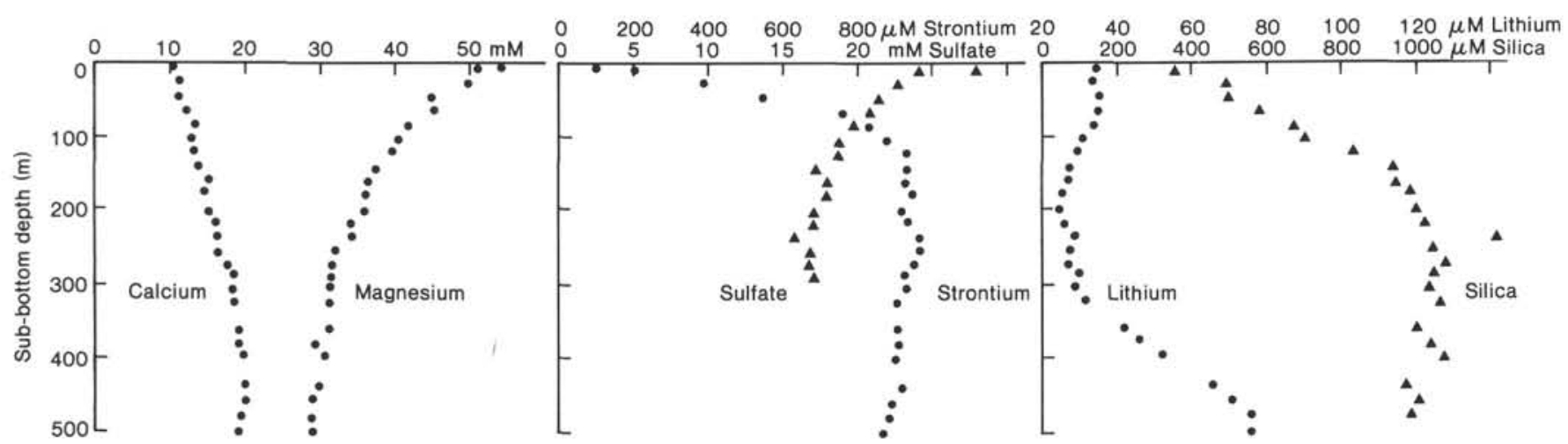

Figure 3. Pore-water concentrations of calcium, magnesium, strontium, sulfate, lithium, and silica as a function of sub-bottom depth at Site 591 . 


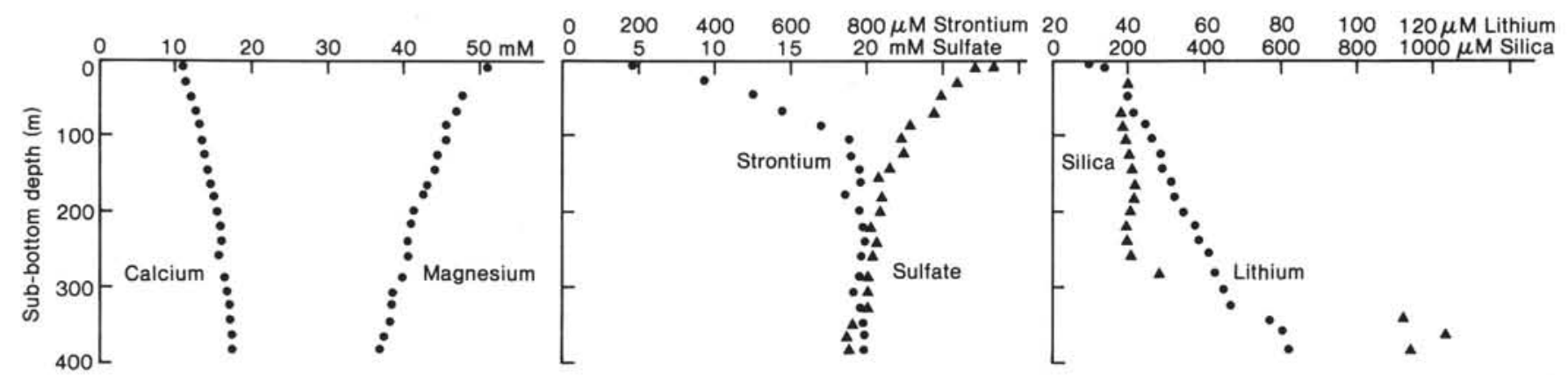

Figure 4. Pore-water concentrations of calcium, magnesium, strontium, sulfate, lithium, and silica as a function of sub-bottom depth at Site 592.

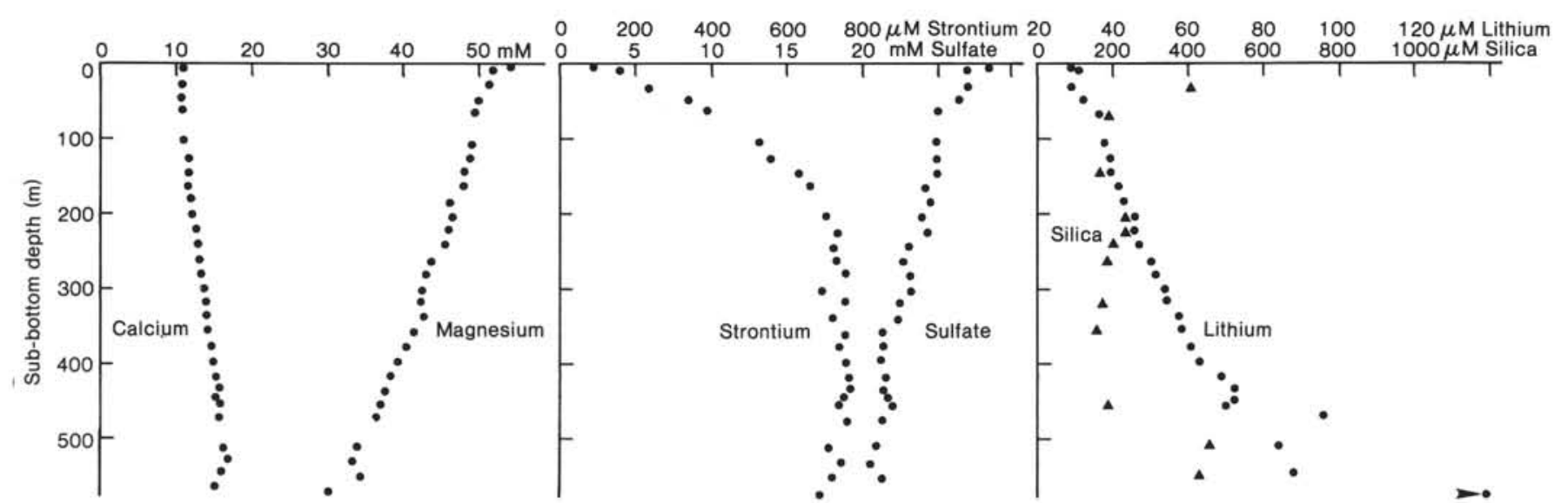

Figure 5. Pore-water concentrations of calcium, magnesium, strontium, sulfate, lithium, and silica as a function of sub-bottom depth at Site 593.

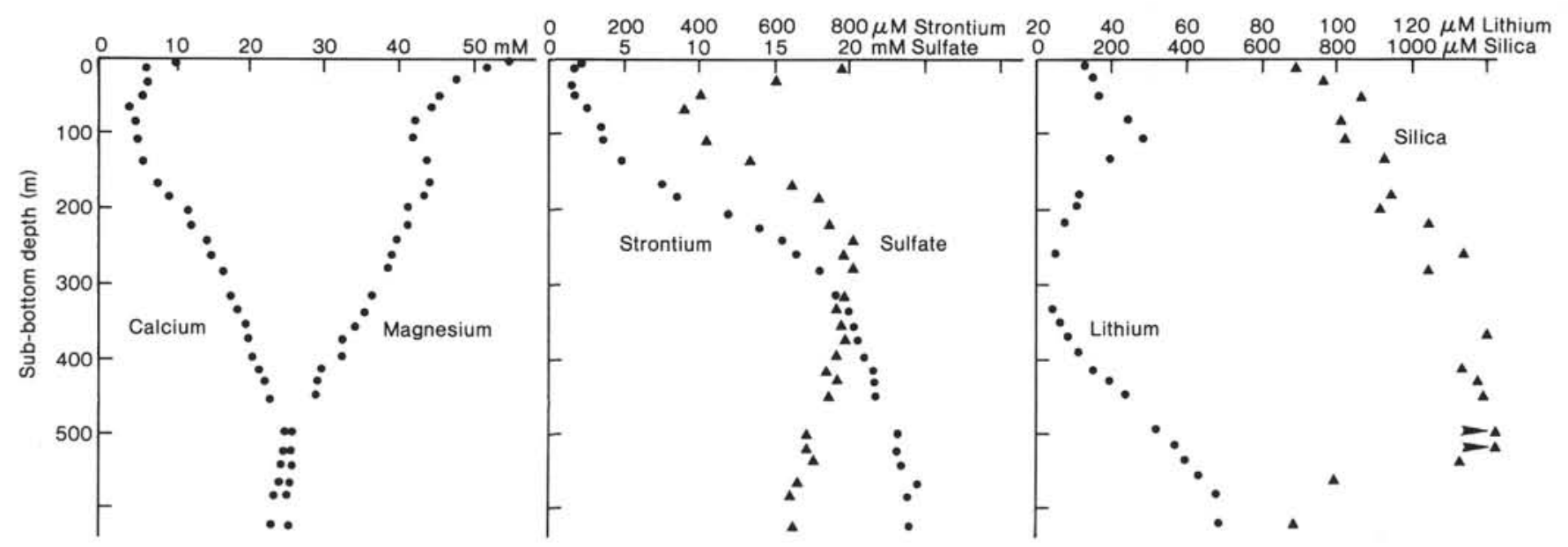

Figure 6. Pore-water concentrations of calcium, magnesium, strontium, sulfate, lithium, and silica as a function of sub-bottom depth at Site 594 .

port for the hypothesis that the entire Lord Howe Rise has an affinity with continental crust (van der Lingen, 1973; Willcox et al., 1980). The minor amount of curvature (toward lower magnesium values) displayed in Figure 7 is consistent with some volcanic ash alteration within the sediment column (Gieskes and Lawrence, 1981), particularly at Sites 590 and 591 .

The alkali metals, lithium and potassium, exhibit trends similar to those observed at many other DSDP sites (e.g., Gieskes, 1983). At all sites except Site 591 there is a slight tendency for potassium concentrations to decrease and lithium concentrations to increase with increasing burial depth. It is clear from the shapes of the lithium concentration-depth curves, that sources and sinks for lithium must occur within the sediment column. At the present time, the nature of these sources and sinks is unknown (Gieskes, 1983).

Dissolved silica concentrations reflect the amount of biogenic silica present in the surrounding sediments (e.g., Gieskes, 1981). Thus, high values of dissolved silica are 


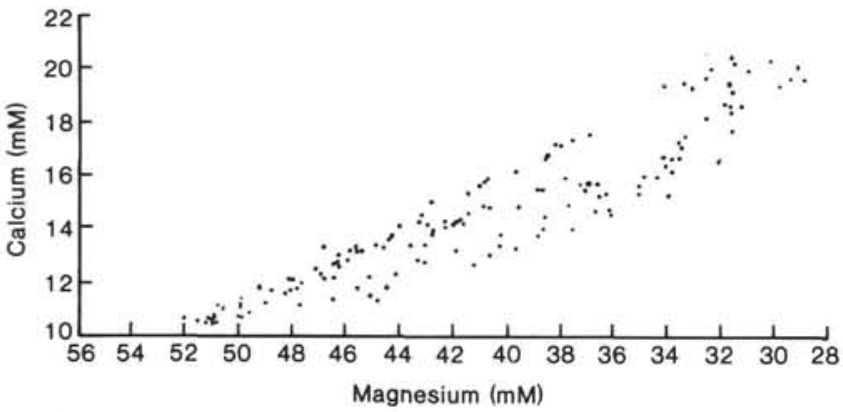

Figure 7. Pore-water concentrations of calcium and magnesium for every sample from the Lord Howe Rise Sites 588, 590, 591, 592, and 593. The data from Sites 590 and 591 lie beneath the general trend.

found at Sites 591 and 594, the two sites that contain the most abundant solid silica. Elsewhere, silica concentrations increase at depth at Sites 590 and 592 . The transformation of biogenic silica to opal-CT is associated with a decrease in dissolved silica as observed at Sites 590, 592, and 594. This "silica front" occurs at depths of about 350,375 , and $550 \mathrm{~m}$ at the respective sites. Chert was recovered at 335 and $380 \mathrm{~m}$ sub-bottom at Site 592, but was not identified at Sites 590 or 594 .

Because of the relatively high rates of carbonate sedimentation, microbial sulfate reduction occurs to some degree at all sites of Leg 90. There is some correlation between the degree of sulfate reduction and the rate of sedimentation, with Sites 590 and 591 having slightly lower sulfate concentrations and higher sedimentation rates than at other sites. Site 594, with its thick hemipelagic Pleistocene section, has a much greater degree of sulfate reduction than any of the Lord Howe Rise sites. At Site 594 an increase in alkalinity accompanies the decrease in sulfate. In turn, the alkalinity increase causes the precipitation of calcium carbonate and a decrease in the $\mathrm{Ca}^{2+}$ concentration.

The strontium concentration profiles are a product of two reactions: carbonate recrystallization and celestite $\left(\mathrm{SrSO}_{4}\right)$ precipitation. As previously shown by Baker et al. (1982), the calcium carbonate tests of foraminifers and coccoliths are enriched in strontium relative to recrystallized calcium carbonate precipitated during burial diagenesis. As a result, there is a net input of strontium to the pore waters of carbonate-rich sediments during the calcite dissolution-reprecipitation reaction. The amount of strontium incorporated into diagenetic carbonate is predicted by the product of the distribution coefficient of strontium in calcite, $\mathrm{k}_{\mathrm{sp}}$ and the ratio of dissolved strontium to dissolved calcium $\mathrm{Sr}^{2+} / \mathrm{Ca}^{2+}$ :

$$
\left(\frac{\mathrm{Sr}}{\mathrm{Ca}}\right)_{\text {calcite }}=\mathrm{k}_{\mathrm{sr}}\left(\frac{\mathrm{Sr}^{2+}}{\mathrm{Ca}^{2+}}\right)
$$

As the amount of recrystallization increases in older and deeper carbonate sediments, the $\mathrm{Sr}^{2+}$ concentration increases, more strontium is incorporated into the reprecipitated calcite, and eventually a steady-state plateau concentration of $\mathrm{Sr}^{2+}$ is achieved.
The $\mathrm{Sr}^{2+}$ concentration at the plateau is typically 0.4 to $0.7 \mathrm{mM}$ in deep-sea carbonate porewaters (Baker et al., 1982). The relatively high sedimentation rates at the Leg 90 sites has led to higher plateau concentrations, ranging from $0.7 \mathrm{mM}$ at Site 588 to $0.9 \mathrm{mM}$ at Sites 591 and 594. As a consequence of these higher concentrations, celestite precipitation occurs. Small nodules of celestite were recovered at Sites 590, 591, and 592. The observed concentration product of strontium sulfate at depth in all the Leg 90 sites averages about $15.2 \times 10^{-6}$ $\mathrm{mol}^{2} / 1^{2}$. The exact value of the $\mathrm{Sr}^{2+}$ concentrations are controlled by celestite precipitation and thus, by the degree of sulfate reduction at each site.

The concentration gradients of $\mathrm{Sr}^{2+}$ versus depth over the upper $100 \mathrm{~m}$ of core at Sites 588, 590, 591, and 592, average $7 \times 10^{-11} \mathrm{~mol} \mathrm{Sr}^{2+} / \mathrm{cm}^{4}$. The average diffusive flux of $\mathrm{Sr}^{2+}$ out of the pore waters at these sites can be calculated simply from Fick's law:

$$
\text { Flux }=-D \frac{d c}{d x}
$$

where $D$ is the diffusion coefficient of $\mathrm{Sr}^{2+}$ in pore waters and $d c / d x$ is the average concentration-depth gradient of $\mathrm{Sr}^{2+}$. Substituting a value of $4 \times 10^{-6} \mathrm{~cm}^{2} / \mathrm{s}$ for $D$, we calculate an average flux of $\mathrm{Sr}^{2+}$ out of the sediments of $2.8 \times 10^{-16} \mathrm{~mol} / \mathrm{cm}^{2}$ per s or $8.8 \times 10^{-3}$ $\mathrm{mol} / \mathrm{cm}^{2}$ per m.y. In a column of sediment that is $100 \mathrm{~m}$ thick, with a porosity of $67 \%$, an average carbonate content of $90 \%$ and an average $\mathrm{Sr} / \mathrm{Ca}$ ratio in the carbonate of $2.0 \times 10^{-3}$ (Baker et al., 1982), there are about

$$
\begin{gathered}
\left(0.9 \times 5 \times 10^{3} \mathrm{~cm}^{3} \mathrm{CaCO}_{3}\left(\frac{2.7 \mathrm{~g} \mathrm{CaCO}_{3}}{\mathrm{~cm}^{3} \mathrm{CaCO}_{3}}\right)\right. \\
\left(\frac{1 \mathrm{~mol} \mathrm{Ca}}{100 \mathrm{~g} \mathrm{CaCO}_{3}}\right)\left(\frac{2.0 \times 10^{-3} \mathrm{~mol} \mathrm{Sr}}{\mathrm{mol} \mathrm{Ca}}\right)
\end{gathered}
$$

$=0.24 \mathrm{~mol} \mathrm{Sr}$ in the solid phase per $\mathrm{cm}^{2}$ of pore water.

The average age of the sediments at $100 \mathrm{~m}$ sub-bottom is about 3 m.y. The diffusive flux of $\mathrm{Sr}^{2+}$ over a 3-m.y. time span amounts to a loss to seawater of $0.026 \mathrm{~mol}$ or $11 \%$ of the total amount of strontium contained in the original sediment. Thus, the minimum rate of recrystallization of the surface sediments is $11 \%$ in $3 \mathrm{~m}$.y. However, much of the strontium released during the dissolution of biogenic calcite is immediately sequestered during the reprecipitation of authigenic calcite. In fact, the "efficiency" of strontium release is probably only about one-third in the upper $100 \mathrm{~m}$ of sediment (and almost zero below $100 \mathrm{~m}$ ). This implies that as much as $30-$ $35 \%$ of the carbonate in the upper $100 \mathrm{~m}$ of sediment is recrystallized!

Values for the distribution coefficient, $\mathrm{k}_{\mathrm{sp}}$, have previously been derived mostly from experimental studies. A limiting value also can be derived in the following manner. The amount of strontium incorporated into authigenic calcite cannot exceed the average amount of stron- 
tium in the biogenic calcite. The latter value, as used, is about $2 \times 10^{-3} \mathrm{~mol} \mathrm{Sr} / \mathrm{mol} \mathrm{Ca}$. At the $\mathrm{Sr}^{2+}$ concentration plateau:

$$
2 \times 10^{-3} \geq \mathrm{k}_{\mathrm{sr}}\left(\frac{\mathrm{Sr}^{2+}}{\mathrm{Ca}^{2+}}\right)_{\text {plateau }}
$$

Using an average value of $5 \times 10^{-2} \mathrm{~mol} / \mathrm{mol}$ for the $\left(\mathrm{Sr}^{2+} / \mathrm{Ca}^{2+}\right)_{\text {plateau }}$, yields a value of $\mathrm{k}_{\mathrm{sr}} \leq 0.04$. This number agrees very well with the results of Katz et al. (1972) and Baker et al. (1982). In the latter paper, we predicted a value of $\mathrm{k}_{\mathrm{sr}} \approx 0.04$ at $5^{\circ} \mathrm{C}$ and $0.08 \mathrm{mM}$ $\mathrm{Sr}^{2+}$.

\section{CONCLUSIONS}

In summary, we can make the following statements on the basis of the observed pore-water chemistry of Leg 90 drill sites. (1) The calcium and magnesium concentration-depth gradients are dominated by silicate alteration reactions occurring mostly at depth beneath the sampled sediments. The unusual value of $\Delta \mathrm{Ca}^{2+} / \Delta \mathrm{Mg}^{2+}$ $\approx-0.45 \mathrm{~mol} / \mathrm{mol}$ is consistent with the observation that the entire Lord Howe Rise is underlain by siliceons rocks. Some volcanic ash alteration can be detected within the sediments at Sites 590 and 591. (2) Minor amounts of microbial sulfate reduction occur at all sites on the Lord Howe Rise. A much higher amount of sulfate reduction occurs in the hemipelagic sediments at Site 594. (3) Strontium pore-water concentrations are determined by carbonate recrystallization reactions. Dissolved strontium concentrations increase downcore until celestite precipitation occurs. At greater depths, strontium concentrations are controlled by thermodynamic equilibrium with respect to celestite, thus Lord Howe Rise sites with lower rates of sulfate reduction have lower concentrations of dissolved strontium. (4) The diffusive flux of strontium in the upper $100 \mathrm{~m}$ of the pore waters can only reasonably be explained by recrystallization of the bulk carbonate sediment at an initial rate of about $10 \% / \mathrm{m}$.y. To verify paleoceanographic conclusions based on isotopic and/ or chemical compositions of the carbonate constituents therefore will require detailed study of the relative reaction rates of different sediment components.

\section{ACKNOWLEDGMENTS}

This work has benefited from detailed reviews by Joris M. Gieskes and Douglas B. Kent. In addition, I thank Paul M. Stout and James V. Gardner for their helpful discussions. Jim Pine extracted all the pore waters aboard the Glomar Challenger. Support was provided for this work by NSF-OCE-8219917.

\section{REFERENCES}

Baker, P. A., Gieskes, J. M., and Elderfield, H., 1982. Diagenesis of carbonates in deep-sea sediments-evidence from $\mathrm{Sr} / \mathrm{Ca}$ ratios and interstitial dissolved $\mathrm{Sr}^{2+}$ data. J. Sediment. Petrol., 52:71-82.
Gieskes, J. M., 1973. Interstitial water studies, Leg 15-alkalinity, pH, $\mathrm{Mg}, \mathrm{Ca}, \mathrm{Si}, \mathrm{PO}_{4}$, and $\mathrm{NH}_{4}$. In Heezen, B. C., MacGregor, I. D., et al., Init. Repts. DSDP, 15: Washington (U.S. Govt. Printing Office), 813-829.

1974. Interstitial water studies, Leg 25. In Simpson, E. S. W., Schlich, R., et al., Init. Repts. DSDP, 25: Washington (U.S. Govt. Printing Office), 361-394.

1981. Deep-sea drilling interstitial water studies: implications for chemical alteration of the oceanic crust, layers I and II. SEPM Spec. Pub., 32:149-167.

1983. The chemistry of interstitial waters of deep sea sediments: interpretation of deep sea drilling data. In Riley, J. P., and Chester, R. (Eds.), Chemical Oceanography (Vol. 8): London (Academic Press), pp. 221-269.

Gieskes, J. M., and Lawrence, J. R., 1976. Interstitial water studies, Leg 35. In Hollister, C. D., Craddock, C., et al., Init. Repts. $D S D P$, 35: Washington (U.S. Govt. Printing Office), 407-423.

1981. Alteration of volcanic matter in deep sea sediments: evidence from the chemical composition of interstitial waters from deep sea drilling cores. Geochim. Cosmochim. Acta, 45:1687-1703.

Gieskes, J. M., Lawrence, J. R., and Galleisky, G., 1978. Interstitial water studies, Leg 38. In Talwani, M., Udintsev, G., et al., Init. Repts. DSDP, Suppl. to Vols. 38, 39, 40, and 41: Washington (U.S. Govt. Printing Office), 121-133.

Katz, A., Sass, E., Starinsky, A., and Holland, H. D., 1972. Strontium behavior in the aragonite-calcite transformation: an experimental study at $40-98^{\circ} \mathrm{C}$. Geochim. Cosmochim. Acta, 36:481-496.

Manheim, F. T., and Sayles, F. L., 1974. Composition and origin of interstitial waters of marine sediments based on deep sea drill cores. In Goldberg, E. D. (Ed.), The Sea (Vol. 5): New York (Wiley-Interscience), 527-568.

Manheim, F. T., Sayles, F. L., and Waterman, L. S., 1972. Interstitial water studies on small core samples, Deep Sea Drilling Project, Leg 12. In Laughton, A. S., Berggren, W. A., et al., Init. Repts. DSDP, 12: Washington (U.S. Govt. Printing Office), 1193-1200.

McDuff, R. E., 1981. Major cation gradients in DSDP interstitial waters: The role of diffusive exchange between seawater and upper oceanic crust. Geochim. Cosmochim. Acta, 45:1705-1713.

McDuff, R. E., and Gieskes, J. M., 1976. Calcium and magnesium in DSDP interstitial waters: diffusion or reaction? Earth Planet. Sci. Lett., 33:1-10.

Presley, B. J., 1971. Techniques for analyzing interstitial water samples. I. Determination of selected minor and major inorganic constituents. In Winterer, E. L., Riedel, W. R., et al., Init. Repts. DSDP, 7, Pt. 2: Washington (U.S. Govt. Printing Office), 1749-1755.

Sandstrom, M., and Gieskes, J. M., 1974. Interstitial water studies, Leg 24. In Fisher, R. L., Bunce, E. T., et al., Init. Repts. DSDP, 24: Washington (U.S. Govt. Printing Office), 799-810.

van der Lingen, G. J., 1973. The Lord Howe Rise rhyolites. In Burns, R. E., Andrews, J. E., et al., Init. Repts. DSDP, 21: Washington (U.S. Govt. Printing Office), 523-540.

Willcox, J. B., Symonds, P. A., Hinz, K., and Bennett, D., 1980. Lord Howe Rise, Tasman Sea-preliminary geophysical results and petroleum prospects. B.M.R. J. Aust. Geol. Geophys., 5:225-236.

Date of Initial Receipt: 18 June 1984 Date of Acceptance: 31 October 1984 